\title{
Originales
}

\section{Eficacia y seguridad de la enoxaparina en la angioplastia primaria. Análisis comparativo con la heparina no fraccionada}

\author{
GUILLERMO GALEOTEa ${ }^{a}$ RAÚL MORENOa , ANGEL SÁNCHEZ-RECALDEa ${ }^{a}$, SANTIAGO JIMÉNEZ-VALEROa \\ LUIS CALVO ${ }^{a}$, FERNANDO RIVERO ${ }^{a}$, JOSÉ F. GALLEGOS ${ }^{b}$, ESTEBÁN LÓPEZ DE SA ${ }^{a}$, JOSÉ A. SOBRINOa \\ Y JOSÉ L. LÓPEZ-SENDÓNa
}

aUnidad de Hemodinámica y Cardiología Intervencionista. Servicio de Cardiología. Hospital Universitario La Paz. Madrid. España.

bUnidad de Bioestadística. Hospital Universitario La Paz. Madrid. España.

Objetivo. El objetivo de este estudio es comparar el efecto de la enoxaparina (ENX) y la heparina no fraccionada (HNF) intravenosas en la angioplastia primaria (AP) del infarto agudo de miocardio (IAMCEST).

Diseño. Estudio prospectivo observacional.

Pácientes y método. Se incluyó en el estudio a 191 pacientes con IAMCEST a los que se había realizado AP. En $91(47,6 \%)$ se utilizó ENX y en $100(52,4 \%)$, HNF. La elección del tratamiento se realizó a criterio del operador. Se excluyó a los pacientes con shock cardiogénico. Los pacientes recibieron un bolo intravenoso de $\operatorname{ENX}(0,75$ o 1 $\mathrm{mg} / \mathrm{kg}$ ) o HNF (70 o $100 \mathrm{U} / \mathrm{kg}$ ), dependiendo si se lo combinaba con abciximab o no. Se realizó seguimiento intrahospitalario evaluando la mortalidad y las complicaciones hemorrágicas de ambos tratamientos.

Resultados. La mortalidad hospitalaria fue del $1,1 \%$ en el grupo ENX y del $3,3 \%$ en el grupo HNF ( $p=0,359)$. Tampoco se encontraron diferencias significativas en el número de complicaciones hemorrágicas con ENX $(4,4 \%)$ y con HNF $(9 \%)$. Hubo 1 trombosis aguda o subaguda de stent con ENX y 3 con HNF. No se encontraron diferencias significativas en el tamaño del infarto medido con troponina I (63,1 con ENX y 54,8 con HNF) ni en la fracción de eyección del ventrículo izquierdo al

Correspondencia: Dr. G. Galeote García.

Servicio de Cardiología. Hospital Universitario La Paz.

$\mathrm{P}^{\circ}$ de la Castellana, 261. 28046 Madrid. España.

Correo electrónico: ggaleote.hulp@ salud.madrid.org

Manuscrito aceptado el 17-9-2008 alta hospitalaria (el $51 \%$ con ENX y el $49,4 \%$ con HNF).

Conclusiones. La AP puede realizarse de forma segura con la administración intravenosa de ENX, pues no se ha encontrado diferencias significativas entre los dos tratamientos ni en mortalidad ni en complicaciones hemorrágicas.

PALABRAS CLAVE: Angioplastia coronaria. Enoxaparina. Stent. Infarto de miocardio.

ENOXAPARIN VS. NON-FRACTIONATED HEPARIN IN PRIMARY ANGIOPLASTY OF ACUTE MYOCARDIAL INFARCTION

Objective. The objective of this study is to compare the effectiveness of intravenous enoxaparin (ENX) and Non-fractionated Heparin (NFH) in primary angioplasty (PA) of acute myocardial infarction (PA-AMI).

Design. A prospective, observational study.

Patients and methods. A total of 191 patients admitted at the hospital with the diagnosis of Acute Myocardial Infarction (AMI) and treated with primary angioplasty were included. ENX was used in 91 patients (47.6\%) and NFH in 100 patients $(52.4 \%)$. Choice of treatment was based on the operator's opinion. Patients with cardiogenic shock were excluded. The first group received an intravenous bolus of ENX $(0.75-1 \mathrm{mg} / \mathrm{Kg})$ and the second one NFH (70-100 $\mathrm{u} / \mathrm{Kg})$, depending on whether it was associated with abciximab or not. In-hospital follow-up was performed, evaluating mortality and bleeding complications of both treatments. 
Results. In-hospital mortality was $1.1 \%$ for the ENX group and $3.3 \%$ for NFH one. No significant differences were found in the number of bleeding complications with ENX $(4.4 \%)$ and NFH $(9.0 \%)$. There was one subacute thrombosis of stent for the ENX group and 3 thrombosis in the NFH-treated group. No significant differences were found in size of infarction measure with troponin I level (63.1 for ENX and 54.8 for the NFH) or in the left ventricle ejection fraction on hospital discharge ( $51 \%$ for ENX and $49.4 \%$ for the NFH).

Conclusions. Primary angioplasty can be safely sued with the intravenous administration of ENX, no significant differences being found between both treatments in mortality and bleeding complications.

KEY WORDS: Coronary angioplasty. Enoxaparin. Stent. Acute myocardial infarction.

\section{INTRODUCCIÓN}

La heparina no fraccionada (HNF) combinada con aspirina (AAS) ha sido el tratamiento antitrombótico clásicamente utilizado en los síndromes coronarios agudos (SCA) y en el intervencionismo coronario percutáneo $(\mathrm{ICP})^{1}$. Sin embargo, la aparición de nuevos fármacos antiplaquetarios (tienopiridinas, inhibidores de la glucoproteína IIb/IIIa) y antitrombóticos como las heparinas de bajo peso molecular (HBPM) ha supuesto la necesidad de estudiar exhaustivamente nuevas estrategias de tratamiento. La HNF tiene algunas limitaciones, como la necesidad de monitorización, una estrecha ventana terapéutica, la potencial inducción de activación plaquetaria y el riesgo de trombocitopenia ${ }^{2}$. Estas limitaciones han hecho que actualmente haya gran interés en valorar nuevos tratamientos anticoagulantes en pacientes sometidos a ICP.

La utilización de HBPM, se ha incrementado en los últimos años en el contexto de SCA e ICP electi$\mathrm{vo}^{3-6}$. Las HBPM tienen ventajas potenciales sobre la HNF, como una más estable y predecible respuesta de la anticoagulación, que obvia la necesidad de monitorización, una mayor vida media y un mayor índice de actividad contra el factor $\mathrm{Xa}$ frente a actividad del factor IIa, el cual reduce la generación y la activación de trombina ${ }^{7}$. Las HBPM inducen menos activación plaquetaria, liberación de factor de von Willebrand e inflamación. En un reciente metaanálisis que comparaba HBPM y HNF en pacientes con SCACEST tratados con trombólisis, De Luca et $\mathrm{al}^{8}$ encontraron una tendencia no significativa a menor mortalidad y una reducción significativa de reinfarto a los 30 días, pero una significativamente mayor proporción de complicaciones hemorrágicas. Esta mayor proporción de complicaciones hemorrágicas se compensaría por las potenciales ventajas de las HBPM.

Actualmente, la AP es el tratamiento de elección en pacientes con infarto agudo de miocardio con elevación del segmento ST (IAMCEST). Las guías de intervencionismo coronario percutáneo (ICP) de la
Sociedad Europea de Cardiología recomiendan que la AP sólo se realice en centros con alta experiencia en ICP y con un programa establecido de tratamiento percutáneo del IAMCEST, siempre que el tiempo puerta-balón sea $\leq 1 \mathrm{~h}$ (recomendación tipo I, nivel de evidencia $\mathrm{A})^{9}$. Se han realizado estudios que comparan HNF con HBPM en pacientes que son sometidos a ICP, pero la información sobre la utilización de HBPM en la AP es muy escasa de momento.

El objetivo de este estudio es evaluar el efecto de dos tipos de tratamiento (ENX y HNF) vía intravenosa en la mortalidad y las complicaciones hemorrágicas de la AP en el contexto de IAMCEST.

\section{PACIENTES Y MÉTODO}

En un periodo comprendido entre enero de 2006 y junio de 2007, se estudió a 206 pacientes consecutivos que se presentaron en nuestro centro con IAMCEST y fueron sometidos a AP. Se realizó un estudio observacional prospectivo en el que la elección del tratamiento que se aplica durante la AP se dejó a criterio del operador. Todos los pacientes tenían dolor torácico de más de 30 min y menos de 12 $\mathrm{h}$ de duración y presentaban en el ECG elevación persistente de ST o bloqueo completo de la rama izquierda del haz de His (BCRIHH).

Se excluyó del estudio a los pacientes que presentaban shock cardiogénico al incio del tratamiento $(6 \%)$, con lo que quedaron como muestra de estudio 191 pacientes. Todos los pacientes, en ausencia de contraindicaciones, recibieron antes de comenzar el procedimiento una dosis de carga intravenosa de ácido acetilsalicílico (AAS) de $500 \mathrm{mg}$ y una dosis de carga vía oral de clopidogrel de $600 \mathrm{mg}$.

En 91 (48\%) se utilizó ENX y en 100 (52\%), HNF. Los pacientes seleccionados para el grupo ENX recibieron un bolo intravenoso de ENX $(0,75$ o $1 \mathrm{mg} / \mathrm{kg})$. Los seleccionados para el grupo HNF recibieron un bolo intravenoso HNF (70 o $100 \mathrm{U} / \mathrm{kg}$ ). La dosis dependía de si se administraba o no el inhibidor de la GPIIb/IIIa abciximab (a criterio del operador). Los pacientes a los que se administró abciximab recibieron un bolo intravenoso de abciximab $(0,25 \mathrm{mg} / \mathrm{kg})$ seguidos de una perfusión intravenosa durante $12 \mathrm{~h}$ $(0,125 \mu \mathrm{g} / \mathrm{kg} / \mathrm{min}$ a un máximo de $10 \mu \mathrm{g} / \mathrm{min})$.

La angioplastia fue realizada de manera estándar por punción percutánea femoral. Se consideró éxito angiográfico cuando se consiguió recanalizar el vaso origen del infarto con un flujo TIMI 2/3. Únicamente se realizó ICP de la arteria obstruida. Se utilizaron dispositivos de cierre percutáneo a discreción del operador. En todos los pacientes se utilizó un introductor de 6 Fr, que fue retirado a las 6 h en la unidad coronaria si no se había utilizado dispositivo de cierre percutáneo en el laboratorio de cateterismo.

\section{Seguimiento clínico}

Los pacientes fueron seguidos durante su ingreso hospitalario tras el procedimiento. En todos los pa- 
cientes se determinaron las máximas concentraciones de creatincinasa $(\mathrm{CK})$, isoenzima $\mathrm{MB}$ de la $\mathrm{CK}(\mathrm{CK}-$ MB) y troponina I tras el infarto agudo de miocardio (IAM). La sensibilidad del test de troponina I fue 0,01 .

Las variables dependientes estudiadas fueron: muerte, reinfarto por trombosis aguda (primeras $24 \mathrm{~h}$ ) o subaguda (desde $24 \mathrm{~h}$ hasta alta hospitalaria) del stent implantado en el vaso tratado y necesidad de nueva revascularización del vaso tratado; las complicaciones hemorrágicas (se consideró hemorragia mayor la que produjese muerte, la de localización intracraneal o intraocular, la caída en la concentración de hemoglobina sérica $>5 \mathrm{~g} / \mathrm{dl} \mathrm{o} \geq 15 \%$ del valor del hematocrito). Se consideró hemorragia menor toda aquella no considerada como mayor y con una caída en la concentración de hemoglobina sérica $<5 \mathrm{~g} / \mathrm{dl}$ (epistaxis, hematemesis, rectorragia, hematoma grave y hematuria macroscópica).

\section{Análisis estadístico}

Los datos se analizaron con el programa estadístico SPSS (v 15.0). Las variables cuantitativas se describen con la media y la desviación típica o con la mediana y el intervalo, según la distribución de los datos. Las variables categóricas se describen con frecuencias absolutas y relativas. Para comparar variables categóricas entre grupos, se utilizó la prueba de la $\chi^{2}$ y el índice de riesgo relativo; para comparar variables cuantitativas entre grupos, se ha utilizado la prueba de la t de Student para muestras independientes. Todas las pruebas estadísticas se han considerado bilaterales y se ha utilizado un nivel de riesgo de 0,05 .

\section{RESULTADOS}

\section{Características clínicas}

Las características clínicas de cada grupo se presentan en la tabla 1. La mayoría de los pacientes eran

\section{TABLA 1. Características clínicas} de ambos grupos

\begin{tabular}{|c|c|c|c|}
\hline Características clínicas & $\begin{array}{l}\text { Enoxaparina } \\
\quad(\mathrm{n}=91)\end{array}$ & $\operatorname{HNF}(\mathrm{n}=100)$ & $\mathrm{p}$ \\
\hline $\begin{array}{l}\text { Tiempo comienzo } \\
\text { dolor-aguja }(\mathrm{h}) \\
\text { media } \pm \mathrm{DE}\end{array}$ & $3,7 \pm 3,8$ & $4,4 \pm 4,4$ & 0,289 \\
\hline $\begin{array}{l}\text { Tratamiento } \\
\quad \text { abciximab, n (\%) }\end{array}$ & $80(87,9)$ & $81(81)$ & 0,190 \\
\hline Varones, $\mathrm{n}(\%)$ & $81(89)$ & $82(82,05)$ & 0,171 \\
\hline Edad, media $\pm \mathrm{DE}$ & $64 \pm 13$ & $61 \pm 15$ & 0,216 \\
\hline HTA, n (\%) & $44(51,8)$ & $50(51,5)$ & 0,977 \\
\hline $\begin{array}{l}\text { Diabetes mellitus, } \\
\mathrm{n}(\%)\end{array}$ & $15(17.6)$ & $25(26)$ & 0,174 \\
\hline Dislipemia, n (\%) & $29(34,1)$ & $35(36,5)$ & 0,742 \\
\hline Tabaquismo, n (\%) & $57(67,1)$ & $64(66)$ & 0,878 \\
\hline CI previa, n (\%) & $8(8,8)$ & $17(17,2)$ & 0,088 \\
\hline IAM anterior & $47(51,6)$ & $41(41,4)$ & 0,364 \\
\hline
\end{tabular}

CI: cardiopatía isquémica; DE: desviación estándar; HTA: hipertensión arterial; IAM: infarto agudo de miocardio. varones en ambos grupos, aunque el sexo masculino fue más frecuente en el grupo ENOX, sin alcanzar significación estadística. No hubo diferencias significativas en el tiempo comienzo del dolor-aguja entre ambos grupos. Los factores de riesgo cardiovascular (FRCV) fueron similares en ambos grupos, excepto en el caso de la diabetes mellitus (DM), con una mayor incidencia (26\%) en el grupo de HNF que tampoco alcanzó significación estadística. No hubo diferencias significativas en la presencia de cardiopatía isquémica $(\mathrm{CI})$ previa definida como IAM, ACTP o bypass previos entre ambos grupos, aunque se encontró en mayor proporción en el grupo HNF (17,2\%). Tampoco hubo diferencias significativas en el tratamiento con abciximab entre ambos grupos (el 87,9\% del grupo ENOX y el $81 \%$ del grupo HNF). Por último, la incidencia de IAM anterior fue similar en ambos grupos.

\section{Características angiográficas}

No hubo diferencias en el tipo de arteria revascularizada entre ambos grupos; la descendente anterior se revascularizó en el 52,7\% del grupo ENX y en el $41 \%$ del grupo HNF; la circunfleja, en el 12,1 y el 15\%; la arteria coronaria derecha, en el 35, 2 y el $43 \%$, respectivamente $(\mathrm{p}=0,339)$. El $42,9 \%$ de los pacientes del grupo ENX y el 39\% del grupo HNF tenían enfermedad coronaria significativa en otras arterias distintas de la obstruida $(\mathrm{p}=0,588)$. La revascularización de otras arterias siempre fue programada y ocurrió mediante ICP en el 10,1\% de los pacientes del grupo ENX y el $13 \%$ del grupo HNF $(p=0,520)$. Un paciente del grupo de HNF se revascularizó mediante cirugía.

El flujo inicial TIMI 2/3 fue del $18 \%$ en el grupo ENX y del $10 \%$ en el grupo HNF ( $p=0,243)$. El flujo TIMI 3 al finalizar el procedimiento fue del $91 \%$ en el grupo de ENX y del $93 \%$ en el grupo de HNF (p $=0,366)$. La tasa de éxito angiográfico de la AP fue del $98,9 \%$ en el grupo de ENX y el $96 \%$ del grupo de $\operatorname{HNF}(\mathrm{p}=0,210)$.

\section{Seguimiento intrahospitalario}

La incidencia de eventos hospitalarios se presenta en la tabla 2. La mortalidad hospitalaria fue del 1,1\% del grupo de ENX y el 3\% del grupo de HNF ( $\mathrm{p}=$

TABLA 2. Incidencia de eventos clínicos intrahospitalarios

\begin{tabular}{|l|c|c|c|}
\hline \multicolumn{1}{|c|}{ Características clínicas } & $\begin{array}{c}\text { Grupo } \\
\text { enoxaparina }\end{array}$ & Grupo HNF & $\mathrm{p}$ \\
\hline Sin complicaciones, n & 86 & 89 & $\mathrm{NS}$ \\
Muerte, n (\%) & $1(1,1)$ & $3(3)$ & 0,359 \\
$\begin{array}{l}\text { Hemorragia, n(\%) } \\
\text { Muerte + hemorragia, } \\
\text { n }(\%)\end{array}$ & $4(4,4)$ & $9(9)$ & 0,207 \\
\hline
\end{tabular}

HNF: heparina no fraccionada. 
0,359). El tamaño del infarto fue similar en ambos grupos (máximo valor de troponina I, $55 \pm 64 \mathrm{UI} / \mathrm{ml}$ en el grupo ENX y $63 \pm 80 \mathrm{UI} / \mathrm{ml}$ en el grupo HNF; $p=0,495)$. La fracción de eyección del ventrículo izquierdo (FEVI) al alta no difirió entre ambos grupos $(51 \% \pm 11 \%$ en el grupo ENX y $49 \% \pm 11 \%$ en el grupo HNF; $\mathrm{p}=0,372)$. La incidencia de complicaciones hemorrágicas, sin alcanzar significación estadística, fue más alta en el grupo de HNF (9\%) que en el grupo ENX (4,4\%; $p=0,207)$. Todos los episodios hemorrágicos mayores se produjeron en el grupo al que se había administrado HNF. Del total de episodios hemorrágicos, 8 se produjeron en el grupo HNF y abciximab, 3 en el grupo ENX y abciximab, 1 en el grupo HNF sola y 1 en el grupo ENX sola. Todos los sangrados en el grupo de ENX fueron menores. Hubo 1 paciente con trombocitopenia severa $(<20.000$ plaquetas) en el grupo de HNF más abciximab. Hubo 1 trombosis aguda o subaguda en el grupo de ENX y 3 en el grupo de HNF, aunque no fue estadísticamente significativo.

\section{DISCUSIÓN}

Nosotros comparamos la seguridad de un bolo intravenoso de ENX con un bolo intravenoso de HNF en la AP, en la mayoría de los casos combinado con abciximab. El hallazgo más importante de nuestro estudio es que la AP puede ser realizada de forma segura utilizando como tratamiento adyuvante ENX combinada con abciximab o no, sin que hayamos encontrado diferencias significativas entre HNF combinada con abciximab y sin combinar en mortalidad ni en complicaciones hemorrágicas. Aún hoy, el fármaco anticoagulante más eficaz en el laboratorio de hemodinámica es objeto de controversia, sobre todo en un escenario tan desfavorable por su ambiente trombótico, como la $\mathrm{AP}^{10}$. Dana et $\mathrm{al}^{11}$ han informado recientemente del hallazgo de trombos macroscópicos en el material de angioplastia (catéteres y guías) en hasta un $5 \%$ de los pacientes a los que se realizó el procedimiento dentro de las $8 \mathrm{~h}$ de la última inyección subcutánea de ENX. Incluso encontraron trombo dentro del catéter guía en un paciente que sufrió una ICP de rescate por trombólisis fallida y que había sido tratado $2 \mathrm{~h}$ antes con bolo intravenoso y subcutáneo de ENX. No hay ensayos clínicos a gran escala que hayan evaluado la ENX en el contexto de AP. Recientemente se han presentado algunos estudios con escaso número de pacientes que evalúan la utilización de ENX en el contexto de AP. En el subestudio del WEST anti-Xa ${ }^{12}$, los 33 pacientes sometidos a AP recibieron, entre otros, ENX subcutánea $1 \mathrm{mg} / \mathrm{kg}$ en el momento del primer contacto médico, pudiendo administrarse un suplemento de ENX intravenosa a dosis de 0,3-0,5 mg/kg en el momento de la AP. En ese estudio se demostró que las dosis de ENX subcutánea previas al procedimiento no fueron eficaces para obtener el rango terapéutico de concentraciones de anti-Xa predeterminado hasta $2 \mathrm{~h}$ después de la administración subcutánea y, por lo tanto, los pacientes no estaban bien anticoagulados en el momento de co- menzar la AP. Esas concentraciones sí se alcanzaron cuando se administraron suplementos intravenosos de ENX. De hecho, el estudio fue diseñado sin suplementos intravenosos de enoxaparina, pero se produjeron tres trombosis de catéter en el grupo inicial, y se cambió la pauta de tratamiento a suplementos intravenosos. Labèque et $\mathrm{al}^{13}$ también han estudiado la administración prehospitalaria de ENX antes de la AP en el IAMCEST. Ese grupo administró un bolo intravenoso de $0,5 \mathrm{mg} / \mathrm{kg}$, seguido de $1 \mathrm{mg} / \mathrm{kg}$ subcutáneo, a nivel prehospitalario antes de la AP. En este estudio prácticamente todos los pacientes tenían adecuados niveles de actividad anti-Xa en el momento de comenzar la AP, aunque se observó excesiva anticoagulación en un $9 \%$ de los pacientes. Nuestro estudio se ha centrado únicamente en pacientes remitidos con SCACEST sometidos a ICP primaria y la administración de ENX fue intravenosa en la sala de hemodinámica. De esta forma no encontramos trombosis asociadas al material utilizado durante el procedimiento y el porcentaje de éxito durante el procedimiento fue muy alto en ambos grupos. Además, nuestros pacientes son pretratados con clopidogrel $600 \mathrm{mg}$ vía oral antes de comenzar el procedimiento con AAS y, si no hay alto riesgo hemorrágico, abciximab. Se utilizó abciximab en más del $80 \%$ de los pacientes de ambos grupos, lo que no fue estadísticamente significativo. La utilización de triple terapia antiplaquetaria más la terapia antitrombótica puede explicar el alto porcentaje de éxito del procedimiento en ambos grupos. El porcentaje de éxito angiográfico de la AP fue del $98,9 \%$ en el grupo ENX y del $96 \%$ en el grupo HNF $(\mathrm{p}=0,210)$.

La ICP primaria es el tratamiento de elección en pacientes con SCACEST, pero por problemas logísticos muchos pacientes todavía son tratados con fármacos trombolíticos. En el registro ARIAM del año 2002, sólo se realizó AP en aproximadamente el 4\% de los pacientes con IAMCEST incluidos en el estudio, con una gran variabilidad entre comunidades autónomas, de las que la Comunidad de Madrid fue donde se utilizó más este tratamiento $(19 \%)^{14}$. La ENX también se ha estudiado en el tratamiento del IAMCEST en combinación con fármacos fibrinolíticos. Théroux et $\mathrm{al}^{15}$ realizaron un metaanálisis de los estudios aleatorizados que comparaban ENX con HNF en combinación con fibrinolíticos en el tratamiento del SCACEST. Este metaanálisis incluyó a 6.069 pacientes y mostró una mejor evolución significativa en pacientes con SCACEST que recibieron enoxaparina en comparación con HNF, como terapia adyuvante a SK, t-PA o tenecteplasa. También hubo un incremento significativo en las tasas de hemorragia grave cuando se usó ENX, sobre todo en combinación con t-PA o tenecteplasa. Sin embargo, en un estudio reciente (el ExTRACT-TIMI 25) ${ }^{16}$ hubo menos eventos isquémicos en el grupo de ENX que con HNF (el 9,9 frente al 12\%; p <0,001), sin diferencias en el número de hemorragias (el 1,4 frente al 1,6\%; $\mathrm{p}$ $=0,56)$. Algunos investigadores creen que la mejor eficacia observada con la ENX en este estudio se debe a un mayor efecto por la prolongación del trata- 
miento ( 7 días con ENX frente a 2 días con $\mathrm{HNF})^{17}$. En un reciente metaanálisis que incluyó el estudio ExTRACT-TIMI 25, el objetivo de eficacia compuesto por muerte e infarto no fatal fue menor con ENX que con HNF (el 9,6 fente al 11,7\%; odds ratio [OR] $=0,78$; intervalo de confianza [IC] del 95\%, 0,67$0,91)$, aunque la incidencia de hemorragia grave fue mayor con ENX que con HNF (el 2,6 frente al 1,8\%; $\mathrm{OR}=0,64 ; \mathrm{IC}$ del $95 \%, 0,52-0,78)^{18}$.

La hemorragia es la complicación más frecuente del tratamiento con heparinas; con la HNF se debe a la inhibición de la coagulación, el daño de la función plaquetaria, el aumento en la permeabilidad capilar y la inducción de trombocitopenia ${ }^{19}$. La HBPM puede producir menos complicaciones hemorrágicas debido a su menor inhibición de la función plaquetaria, que no incrementa la permeabilidad capilar y porque induce trombocitopenia con menor frecuencia ${ }^{20,21}$. Borentain et $\mathrm{al}^{6}$ realizaron un metaanálisis de los estudios aleatorizados y no aleatorizados que comparaban la HBPM con la HNF en el ICP, usada de forma intravenosa en el laboratorio de cateterismo en pacientes previamente no tratados con alguna forma de anticoagulante. En ese metaanálisis se incluyó a 3.787 pacientes que recibieron HBPM intravenosa en el contexto de ICP electivo. El objetivo combinado de eficacia fue el 5,8 frente al 7,6\% ( $\mathrm{p}=0,03)$, hemorragia mayor del 0,6 frente al $1,8 \%(\mathrm{p}=0,0001)$, hemorragia menor del 3,1 frente al 3,1\%, todas las hemorragias el 3,7 frente al 4,9\% cuando se comparaba HBPM vs HNF; es decir, que hubo menos hemorragias graves en el grupo de HBPM, aunque no hubo diferencias significativas en el total de hemorragias. La enoxaparina fue la HBPM más usada en estos estudios $(\mathrm{n}=3.075)$. Es verdad que en el metaanálisis de exclusivamente los 8 estudios aleatorizados ${ }^{22-25}$ no se encontraron diferencias significativas entre los dos tipos de tratamiento, pero sí una fuerte tendencia a que la utilización de HBPM intravenosa sin monitorizar en el laboratorio de cateterismo era tan segura y eficaz como la utilización de HNF con monitorización de TCA, esta tendencia también se observó en menos hemorragias en el grupo de HBPM. Sin embargo, cuando se incluyeron en el análisis los 7 estudios no aleatorizados ${ }^{5,26-29}$, sí se encontraron diferencias a favor de la utilización de las HBPM. El problema es que ninguno de los 15 estudios utilizados en este metaanálisis tenía poder estadístico adecuado para obtener conclusiones definitivas. También se analizaron los diferentes tipos de dosis de ENX utilizadas en los diferentes estudios, y se encontró que una dosis baja de $0,5 \mathrm{mg} / \mathrm{kg}$ se relacionó con pocos eventos coronarios y aún menos hemorragias, independientemente de que se combinara con anti-GPIIb/ IIIa o no.

Con objeto de despejar estas dudas se diseñó el estudio Safety and Efficacy of Enoxaparin in PCI Patients, an International Randomized Evaluation (STEEPLE) trial ${ }^{30}$. Este estudio evaluó dos dosis de ENX, 0,5 o 0,75 mg/kg, comparadas con HNF en pacientes sometidos a ICP electivo. Se incluyó en el estudio a 3.528 pacientes. Hubo hemorragia mayor o menor durante las primeras $48 \mathrm{~h}$ en el 5,9\% de los pacientes del grupo de $0,5 \mathrm{mg}$, en el $6,5 \%$ en el grupo de $0,75 \mathrm{mg}$ y en el $8,5 \%$ del grupo de HNF. Estos valores representan una reducción relativa del $31 \%$ en la aparición de hemorragias en el grupo de $0,5 \mathrm{mg} /$ $\mathrm{kg}$ de ENX respecto a $\mathrm{HNF}(\mathrm{p}=0,01)$ y una reducción relativa del $24 \%$ en el grupo de $0,75 \mathrm{mg} / \mathrm{kg}$ respecto a $\mathrm{HNF}(\mathrm{p}=0,051)$. Otro estudio reciente que comparó de ENX con HNF en pacientes con SCA de alto riesgo sometidos a ACTP es el ensayo SYNERGY ${ }^{31}$. En ese estudio la administración de ENX o HNF era previa a la realización de la ACTP, y no se administraba más ENX en el laboratorio si la última dosis subcutánea se había dado menos de $8 \mathrm{~h}$ antes. No hubo diferencias en el procedimiento ni en la tasa de eventos isquémicos entre los dos grupos. Tampoco hubo diferencias en la incidencia de hemorragias. Los resultados de estos estudios son congruentes con los hallazgos obtenidos en nuestro trabajo, si bien en éste todos los pacientes fueron sometidos a AP y la administración de la ENX fue en bolo intravenoso en la sala de hemodinámica, sin administración previa de heparina subcutánea. En nuestro estudio la incidencia total de complicaciones hemorrágicas (sin alcanzar significación estadística) fue más alta en el grupo de HNF (9\%) que en el grupo de enoxaparina $(4,4 \% ; \mathrm{p}=0,207)$, muy similar a la comunicada en el estudio STEEPLE, en los grupos de enoxaparina $0,75 \mathrm{mg}$ y $\mathrm{HNF}$, entre los que tampoco hubo diferencias significativas. Además, en la mayoría de nuestros pacientes en ambos grupos se administró abciximab, que es un grupo de alto riesgo hemorrágico. Nuestra tasa de hemorragias fue del 4,3\% en el grupo tratado con ENX y el $14,5 \%$ en el grupo tratado con HNF; si lo comparamos con los resultados del estudio STEEPLE, el número de hemorragias en nuestro grupo de ENX es inferior al de su grupo de $0,75 \mathrm{mg} / \mathrm{kg}+$ abciximab $(10,8 \%)$ y muy similar en el grupo HNF $(11,2 \%)$.

Se ha discutido ampliamente sobre la dosis más adecuada de utilización de ENX en el contexto de ACTP. La dosis es importante porque hasta hace poco no existía un método sencillo de valoración del efecto de este fármaco en la anticoagulación a la cabecera del paciente, al contrario que con la HNF. En nuestro estudio hemos usado las dosis de $0,75 \mathrm{mg} / \mathrm{kg}$ intravenosa cuando agregábamos abciximab y de $1 \mathrm{mg} / \mathrm{kg}$ intravenosa si no lo agregábamos. Estas dosis han sido las recomendadas desde los primeros estudios NICE en el contexto de ICP y nuestro grupo tiene amplia experiencia, con buenos resultados y niveles estables de actividad anti-Xa. Algunos autores han comunicado buenos resultados con menor tasa de eventos hemorrágicos utilizando la dosis intravenosa de $0,5 \mathrm{mg}$, y en el estudio STEEPLE fue la dosis que mostró significativamente menor número de eventos hemorrágicos respecto a la HNF. En este estudio los niveles de anticoagulación diana se alcanzaron en un $79 \%$ de los pacientes del grupo con $0,5 \mathrm{mg}$ y en un $92 \%$ de los pacientes del grupo $0,75 \mathrm{mg}$; sin embargo, en este estudio se excluyó a los pacientes sometidos a AP y, además, en el grupo de $0,5 \mathrm{mg}$ el reclutamiento 
se terminó precozmente por una excesiva mortalidad, no claramente explicada.

Por último, la trombocitopenia es más frecuente con la HNF que con la HBPM. En nuestro estudio hubo 1 paciente con trombocitopenia severa en el grupo HNF con abciximab.

\section{Limitaciones del estudio}

Este estudio es observacional y prospectivo. La utilización del tratamiento se dejó a criterio del operador y no se aleatorizó, reflejando lo que ocurre en el mundo real, pero pudo ocurrir algún sesgo de selección, aunque esto probablemente no influyó en los eventos hemorrágicos posteriores. Se han publicado recientemente estudios aleatorizados que avalan la utilización de la estrategia ENX con o sin abciximab en la ICP electiva o urgente, pero no se ha estudiado en el contexto de la AP; por ello sería necesario realizar estudios aleatorizados en este contexto para extraer conclusiones definitivas.

\section{CONCLUSIONES}

La AP puede ser realizada de forma segura utilizando como tratamiento adyuvante ENX combinada con abciximab o no en administración intravenosa, sin diferencias significativas en la incidencia de muerte y complicaciones hemorrágicas entre ambos tipo de tratamiento.

\section{BIBLIOGRAFÍA}

1. Antiplatelet Trialists Collaboration. Collaborative overview of randomised trials of antiplatelet therapy. Prevention of death myocardial infarction and stroke by prolonged antiplatelet therapy in various categories of patients. BMJ. 1994;308:81-106.

2. Cohen M. The role of low-molecular-weight heparin in the managegement of acute coronary syndromes. J Am Coll Cardiol 2003;41 Suppl 4:S55-61.

3. Ferguson JJ, Antman EM, Bates ER, Cohen M, Every NR, Harrington RA, et al. Combining enoxaparin and glycoprotein IIb/IIIa antagonists for the treatment of acute coronary syndromes: final results of the National Investigators Collaborating on Enoxaparin-3 (NICE-3) study. Am Heart J. 2003;146:628-34.

4. Ferguson JJ, Califf RM, Antman EM, Cohen M, Grines CL, Goodman S, et al. Enoxaparin vs unfractionated heparin in highrisk patients with non-ST-segment elevation acute coronary syndromes managed with an intended early invasive strategy: primary results of the SYNERGY randomized trial. JAMA. 2004;292: 45-54.

5. Choussat R, Montalescot G, Collet JP, Vicaut E, Ankri A, Gallois V, et al. A unique, low dose of intravenous enoxaparin in elective percutaneous coronary intervention. J Am Coll Cardiol. 2002;40:1943-50.

6. Borentain M, Montalescot G, Bouzamondo A, Choussat R, Hulot JS, Lechat P. Low-molecular-weight heparin vs. unfractionated heparin in percutaneous coronary intervention: a combined analysis. Catheter Cardiovasc Interv. 2005;65:212-21.

7. Samama MM, Gerotziafas GT. Comparative pharmacokinetics of LMWHs. Semin Thromb Hemost. 2000;26 Suppl $1: 31-8$.

8. De Luca G, Marino P. Adjunctive benefits from low-molecular-weight heparins as compared to unfractionated heparin among patients with ST-segment elevation myocardial infarction treated with thrombolysis. A meta-analysis of the randomized trials. Am Heart J. 2007;154:1085.e1-6.

9. Silber S, Albertsson P, Avilés FF, Camici PG, Colombo A, Hamm C, et al; Task Force for Percutaneous Coronary Interventions of the European Society of Cardiology. Guidelines for percutaneous coronary interventions. Eur Heart J. 2005;26:804-47.

10. Montalescot G, Cohen M. Low molecular weight heparins in the cardiac catheterization laboratory. J Thromb Thrombolysis. 1999; 7:319-23.

11. Dana A, Nguyen CM, Cloutier S, Barbeau GR. Macroscopic thrombus formation on angioplasty equipment following antithrombin therapy with enoxaparin. Catheter Cardiovasc Interv. 2007; $70: 847-53$

12. Welsh RC, Gordon $P$, Westerhout CM, Buller CE, O'neill B, Armstrong PW. A novel enoxaparin regime for ST elevation myocardial infarction patients undergoing primary percutaneous coronary intervention: A WEST sub-study. Catheter Cardiovasc Interv. 2007;70:341-8

13. Labeque JN, Jais C, Dubos O, Denard M, Berhouet M, Leroux L, et al. Prehospital administration of enoxaparin before primary angioplasty for ST-elevation acute myocardial infarction. Catheter Cardiovasc Interv. 2006;67:207-13

14. Colmenero Ruiz M, Reina Toral A, Jiménez Quintana MM, Aguayo de Hoyos E, Ruiz Bailén M, García Alcántara A, et al; Grupo ARIAM. Variabilidad en el manejo del infarto agudo de miocardio en el registro ARIAM en el año 2002. Med Intensiva. 2005;29:420-9.

15. Theroux $P$, Welsh RC. Meta-analysis of randomized trials comparing enoxaparin versus unfractionated heparin as adjunctive therapy to fibrinolysis in ST-elevation acute myocardial infarction. Am J Cardiol. 2003;91:860-4.

16. Antman EM, Morrow DA, McCabe $\mathrm{CH}$, Murphy SA, Ruda M, Sadowski Z, et al. Enoxaparin versus unfractionated heparin with fibrinolysis for ST-elevation myocardial infarction. N Engl J Med. 2006;354:1477-88.

17. Comparación de enoxaparina y heparina no fraccionada en el infarto agudo de miocardio tratadado con fibrinólisis. Med Intensiva. 2006;30:350-3.

18. Murphy SA, Gibson CM, Morrow DA, Van de Werf F, Menown IB, Goodman SG, et al. Efficacy and safety of the lowmolecular weight heparin enoxaparin compared with unfractionated heparin across the acute coronary syndrome spectrum: a metaanalysis. Eur Heart J. 2007;28:2077-86.

19. Weitz JI. Low-molecular-weight heparins. N Engl J Med. 1997;337:688-98.

20. Zed PJ, Tisdale JE, Borzak S. Low-molecular-weight heparins in the management of acute coronary syndromes. Arch Intern Med. 1999;159:1849-57.

21. Warkentin TE, Levine MN, Hirsh J, Horsewood P, Roberts RS, Gent M, et al. Heparin-induced thrombocytopenia in patients treated with low-molecular-weight heparin or unfractionated heparin. N Engl J Med. 1995;332:1330-5.

22. Karsch KR, Preisack MB, Baildon R, Eschenfelder V, Foley D, Garcia EJ, et al. Low molecular weight heparin (reviparin) in percutaneous transluminal coronary angioplasty. Results of a randomized, double-blind, unfractionated heparin and placebo-controlled, multicenter trial (REDUCE trial). Reduction of Restenosis After PTCA, Early Administration of Reviparin in a Double-Blind Unfractionated Heparin and Placebo-Controlled Evaluation. J Am Coll Cardiol. 1996;28:1437-43.

23. Rabah MM, Premmereur J, Graham M, Fareed J, Hoppensteadt DA, Grines LL, et al. Usefulness of intravenous enoxaparin for percutaneous coronary intervention in stable angina pectoris. Am J Cardiol. 1999;84:1391-5.

24. Bhatt DL, Lee BI, Casterella PJ, Pulsipher M, Rogers M, Cohen M, et al. Safety of concomitant therapy with eptifibatide and enoxaparin in patients undergoing percutaneous coronary intervention: results of the Coronary Revascularization Using Integrilin and Single bolus Enoxaparin Study. J Am Coll Cardiol. 2003;41:20-5.

25. Galeote G, Hussein M, Sobrino N, Calvo L, SanchezRecalde A, Sobrino JA. Utilización de enoxaparina o heparina no fraccionada en combinación con abciximab durante la intervención coronaria percutánea: estudio piloto aleatorizado. Rev Esp Cardiol. 2002;55:1261-6. 
26. Kereiakes DJ, Kleiman NS, Fry E, Mwawasi G, Lengerich $\mathrm{R}$, Maresh K, et al. Dalteparin in combination with abciximab during percutaneous coronary intervention. Am Heart J. 2001;141: $348-52$.

27. Kereiakes DJ, Grines C, Fry E, Esente P, Hoppensteadt D, Midei M, et al. Enoxaparin and abciximab adjunctive pharmacotherapy during percutaneous coronary intervention. J Invasive Cardiol. 2001;13:272-8.

28. Chen WH, Lau CP, Lau YK, Ng W, Lee PY, Yu CM, et al. Stable and optimal anticoagulation is achieved with a single dose of intravenous enoxaparin in patients undergoing percutaneous coronary intervention. J Invasive Cardiol. 2002;14:439-42.

29. Miller L, Gupta A, Bertolet BD. Use of clopidogrel loading, enoxaparin, and double-bolus eptifibatide in the setting of early percutaneous coronary intervention for acute coronary syndromes. J Invasive Cardiol. 2002;14:247-50.

30. Montalescot G, White HD, Gallo R, Cohen M, Steg PG, Aylward PE, et al. Enoxaparin versus unfractionated heparin in elective percutaneous coronary intervention. N Engl J Med. 2006; $355: 1006-17$

31. White HD, Kleiman NS, Mahaffey KW, Lokhnygina Y, Pieper KS, Chiswell K, et al. Efficacy and safety of enoxaparin compared with unfractionated heparin in high-risk patients with non-ST-segment elevation acute coronary syndrome undergoing percutaneous coronary intervention in the Superior Yield of the New Strategy of Enoxaparin, Revascularization and Glycoprotein IIb/IIIa Inhibitors (SYNERGY) trial. Am Heart J. 2006;152 1042-50. 